\title{
A Multilevel Tailored Web App-Based Intervention for Linking Young Men Who Have Sex With Men to Quality Care (Get Connected): Protocol for a Randomized Controlled Trial
}

José A Bauermeister ${ }^{1}, \mathrm{MPH}, \mathrm{PhD}$; Jesse M Golinkoff ${ }^{1}$, MPH; Keith J Horvath ${ }^{2}$, PhD; Lisa B Hightow-Weidman ${ }^{3}$, MD, MPH; Patrick S Sullivan ${ }^{4}$ DVM, PhD; Rob Stephenson ${ }^{5}$, PhD

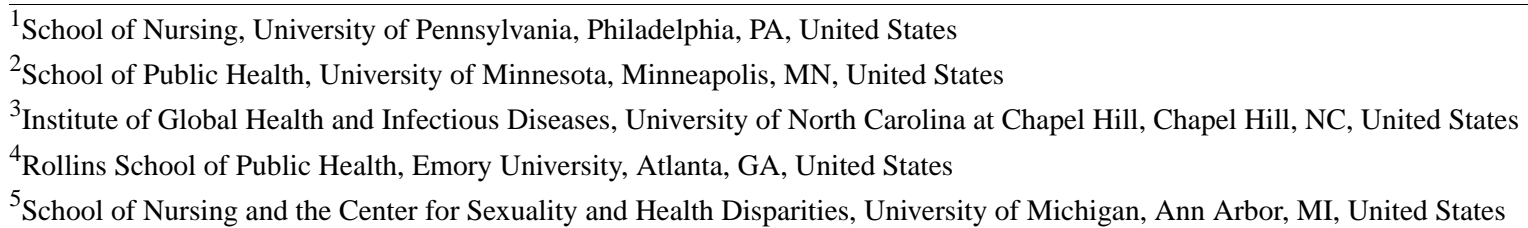

\section{Corresponding Author:}

José A Bauermeister, MPH, PhD

School of Nursing

University of Pennsylvania

418 Curie Boulevard

Suite 402

Philadelphia, PA, 19109

United States

Phone: 1 (215) 8989993

Fax: 1 (215) 7463374

Email: bjose@upenn.edu

\section{Abstract}

Background: HIV epidemic among young men who have sex with men (YMSM) is characterized by strong racial disparities and concerns about the availability and access to culturally appropriate HIV prevention and care service delivery. Get Connected, a Web-based intervention that employs individual- and system-level tailoring technology to reduce barriers to HIV prevention care (eg, HIV or sexually transmitted infection [STI] testing, pre-exposure prophylaxis [PrEP]), was developed for YMSM (age 15-24 years). This protocol details the design and procedures of a 2-phase project that includes mystery shopping and a randomized controlled trial (RCT) to test the efficacy of Get Connected among YMSM in Philadelphia, Atlanta, and Houston.

Objective: The objective of mystery shopping is to examine the quality of HIV test counseling and PrEP-related referrals for YMSM within local HIV or STI testing sites. The objective of the RCT is to test the efficacy of Get Connected for increasing HIV-negative or HIV-unknown YMSM's successful uptake of HIV prevention services (eg, routine HIV or STI testing), PrEP awareness, and likelihood to start PrEP (PrEP willingness), compared with those in the control condition, over a 12-month period.

Methods: For Phase 1, we will create a master list of HIV and STI testing sites in each city. We will enroll and train 10-15 mystery shoppers per city; each testing site will be separately visited and assessed by 2 mystery shoppers. After each site visit, the mystery shoppers will complete a site evaluation to record their perceptions of various measures including lesbian, gay, bisexual, transgender, queer visibility and inclusivity, privacy and confidentiality, provider-patient interactions, and clinic environment. For Phase 2, we will enroll 480 YMSM for 12 months across the 3 iTech cities into a 2-arm prospective RCT. Participants randomized to the control condition are directed to the AIDSVu.org testing site locator. Participants randomized to the intervention condition will be granted access to a Web app with content tailored to their specific demographic characteristics (eg, age, race or ethnicity, location, and relationship status), HIV and STI risk behaviors (eg, HIV and STI testing history, substance use, communication with partners regarding status) and sociocultural context (eg, homelessness, incarceration). Study assessments will occur at enrollment and at 1, 3, 6, 9, and 12 months postenrollment.

Results: Get Connected research activities began in September 2016 and are ongoing. To date, institutional review board (IRB) submission is complete and IRB authorization agreements are pending at several other universities. 
Conclusions: The deployment of Get Connected through a mobile-optimized Web app seeks to optimize the intervention's acceptability, accessibility, availability, and long-term affordability among YMSM.

Trial Registration: ClinicalTrials.gov (NCT03132415); https://clinicaltrials.gov/ct2/show/NCT03132415 (Archived by WebCite at http://www.webcitation.org/70j4gSFbZ)

Registered Report Identifier: RR1-10.2196/10444

(JMIR Res Protoc 2018;7(8):e10444) doi: 10.2196/10444

\section{KEYWORDS}

HIV infections; AIDS; adolescent; internet; men who have sex with men; prevention; pre-exposure prophylaxis; awareness

\section{Introduction}

\section{Background}

Young men who have sex with men (YMSM) now account for $72 \%$ of new HIV infections among people aged 13-24 years and $30 \%$ of all new infections among men who have sex with men (MSM) [1]. From 2008 to 2011, YMSM aged 13-24 years had the greatest percentage increase $(26 \%)$ in diagnosed HIV infections [2], with approximately $93 \%$ of all diagnosed HIV infections from male-to-male sexual contact [2]. Among the drivers of the HIV epidemic among YMSM are large numbers of HIV-positive youth who are not virally suppressed or are not aware of their serostatus [3]. Increasing HIV testing among YMSM is thus a public health priority [4]. The success of the National HIV/AIDS Strategy's test and treat approach rests on the ability to increase the number of YMSM receiving routine testing [5]. Getting tested is the cornerstone of almost all prevention approaches and the gateway to both biomedical prevention tools (eg, pre-exposure prophylaxis [PrEP]) and to HIV care for those who test positive.

Successful engagement in HIV prevention for HIV-negative youth (routine HIV testing, consistent condom use, PrEP adoption) requires that YMSM overcome a series of multilevel barriers at the individual (eg, risk awareness, self-efficacy to get tested), system (eg, costs, medical mistrust, lack of culturally competent care), and structural (eg, homelessness, stigma) levels [6-12]. Strategies to promote HIV or sexually transmitted infection (STI) status awareness among YMSM requires the creation of interventions that are culturally sensitive to the psychosocial needs of YMSM [13] and facilitate access to comprehensive sexual health services [14].

HIV prevention tools that are culturally and developmentally appropriate for YMSM are needed $[1,7,15,16]$. Web-based interventions are a promising mode of HIV or STI prevention given their ability to deliver responsive and interactive content specific to each user's characteristics (ie, tailored content), with extended reach across geographic regions and increased convenience to access content at any time through tablets, laptops, and mobile phones. Furthermore, Web-based content can be updated to be contextually responsive over time, particularly as YMSM become sexually active, meet new partners, and engage in different risk behaviors. Collocating Web-based interventions is also important as YMSM often rank the Web as their top resource to access comprehensive sexual education, learn about their sexuality and sexual behavior, and meet partners [17].
Researchers and practitioners have sought to encourage routine HIV or STI testing by creating Web-based tools that provide the physical location of testing centers in a given geographic area (ie, testing locators). These testing locator interventions have demonstrated a wide reach when evaluated (eg, AIDS.gov test locator had over 16,000 searches and was adopted by over 100 websites in its first year [18]); however, there are limited data examining the quality and adequacy of these listed sites for YMSM. This is concerning for several reasons, as it is expected that testing agencies are youth and lesbian, gay, bisexual, transgender, questioning or queer (LGBTQ) friendly; however, there is little empirical evidence to support this assumption, and in fact evidence to support the contrary [19-23]. Using a mystery shopper methodology to evaluate the LGBTQ cultural competency and the quality of services offered in HIV and STI testing sites in Southeast Michigan ( $\mathrm{n}=47$ testing sites), we assessed the sites across 13 domains, including the clinic's structural characteristics, and the test counselors' compliance with the Centers for Disease Control and Prevention (CDC) HIV testing and counseling protocols [6]. After the mystery shopping assessment, we sent each site a letter describing our process and encouraged them to schedule a meeting with us to discuss the shoppers' experiences at their agency. The agency staff was eager for the feedback and technical assistance, and $66 \%$ (31/47) of the sites requested to meet. In these meetings, we offered a packet of personalized results, summarizing how they compared on various quantitative indicators to other sites, and provided feedback from the open-ended portion of the evaluation. Several agencies noted that the report from the site evaluation would help focus their efforts and address identified areas for improvement.

\section{Theoretical Framework for Intervention}

Building on the efficacy of the CDC's project Connect Health Systems Intervention to link heterosexual adolescents to competent comprehensive sexual health care services [24], we developed Get Connected (GC), a Web-based brief intervention that employs individual- and system-level tailoring technology to reduce barriers to HIV prevention care (eg, HIV or STI testing, PrEP) for YMSM. The deployment of GC through a mobile-friendly Web app seeks to optimize the Web-based intervention's acceptability, accessibility, availability, and long-term affordability among youth $[4,7,25]$. Using a consensus approach [26] to conceptualize health behavior change, the model guiding GC synthesizes the Integrated Behavioral Model [27] and Self-Determination Theory [28,29] as the theoretical underpinnings of our intervention. Consistent with these theories [30,31], GC content follows motivational interviewing principles 
[31-33] by focusing on resolving ambivalence about HIV prevention behaviors, increasing self-efficacy for change, and enhancing motivation moving toward action. GC participants are then recommended high-performing sites based on mystery shopper scores.

Participants in the pilot trial were randomized to receive a full GC intervention or an attention-control condition. Data [34] from this pilot randomized controlled trial (RCT; $n=130$ YMSM; age 15-24 years) indicated high acceptability and feasibility (80\% retention, 104/130) for GC, and clinically meaningful effect sizes (ES) in self-efficacy to discuss HIV testing with partners $(\mathrm{ES}=0.50-0.64)$, trust in their providers $(\mathrm{ES}=0.33-0.35)$, reductions in number of sexual partners $(\mathrm{ES}=0.21)$, and HIV or STI testing behavior (ES=0.34; 30 participants tested for HIV or STIs) at the 30-day postintervention follow-up. Participants who received the GC intervention reported that the testing site information was more accurate than those in control condition. For all other acceptability items, the GC intervention was equally or slightly better received than the control condition. More than $90 \%(102 / 104,92.3 \%)$ of participants reported that the GC intervention had been useful to identify a HIV or STI clinic that met their needs. We identified 1 incident HIV-positive case and 2 STI (herpes and chlamydia) cases over the 30-day study period.

As a step toward filling the current gap in efficacious Web-based interventions for HIV prevention and care among YMSM, we propose to implement and test the efficacy of GC 2.0 across 3 iTech cities heavily impacted by HIV: Philadelphia, Atlanta, and Houston. This protocol describes the methods for the testing of the intervention.

\section{Methods}

\section{Trial Registration, Ethics, Consent, and Institutional Review Board Approval}

The research and ethics presented in this study were approved by the IRB of the University of North Carolina at Chapel Hill (16-3183). A Certificate of Confidentiality has been obtained from the National Institute of Child Health and Human Development, and a waiver of parental consent or assent has been obtained for participants who are 15-17 years old. This study is also registered on ClinicalTrials.gov (NCT03132415).

\section{Phase 1: Mystery Shopping}

\section{Design}

We will enroll mystery shopping participants (10-15 mystery shoppers per city) to conduct the mystery shopper assessment in 3 iTech cities: Philadelphia, Atlanta, and Houston. This approach follows best practices suggesting that youth involvement is vital when designing relevant and appropriate HIV interventions for the target population. We will work with iTech subject recruitment venues (SRV) in each city to recruit and enroll HIV-negative YMSM (age 18-24 years) who are interested in serving as mystery shoppers. We will apply a multimodal recruitment strategy, including ads in Web-based LGBTQ listservs, flyers in HIV or AIDS community-based organizations, local coffee shops and bars, college listservs, and
Web-based advertisements on social media sites such as Facebook.

\section{Mystery Shopper Participants}

Eligible mystery shoppers are participants assigned male sex at birth and who currently identify as male; must be 18-24 years old (inclusive) at the time of screening; self-report as HIV-negative, speak and read English, live in Philadelphia, Atlanta, or Houston; must be able to travel to and from HIV or STI testing sites; report same-sex attraction; and have access to the internet via a computer or mobile phone.

\section{Sample Size}

We will recruit and enroll 10-15 mystery shoppers per city. Each participant can visit up to 10 unique testing sites in their city, with 2 mystery shoppers visiting each testing site, separately. Testing sites will be identified in collaboration with each city's health department and by crosschecking sites with AIDSVu.org. We will employ a stratified purposive sampling strategy to ensure age and racial or ethnic diversity across mystery shoppers. Of the 10-15 mystery shoppers per city, 5-8 will be aged 18-20 years (2-3 Black or African American, 2-3 White, and 1-2 Hispanic or Latino) and 5-8 will be aged 21-24 years (2-3 Black or African American, 2-3 White, and 1-2 Hispanic or Latino).

\section{Incentives}

The mystery shoppers will receive a maximum of US \$600: US $\$ 100$ for attending the 1-day training session and US $\$ 50$ for each testing site visit (10 maximum site visits).

\section{Procedures}

Once the mystery shoppers are consented and enrolled, they will attend a 1-day training at the iTech SRV where they will learn about the fundamentals of HIV or STI transmission, the guidelines and protocols surrounding HIV or STI testing and PrEP eligibility, and how to use the Web-based site assessment survey to evaluate their site visits. State-specific guidelines and policies will also be discussed in each city. Additionally, they will receive training to strengthen their self-efficacy to feel empowered as clients. Specifically, we will conduct role-plays with scenarios and interactions that might occur during a visit. We will underscore the importance of being well-versed in their rights and procedures and provide skills on how to respond to worst-case scenarios (eg, how to turn down any unwanted procedures), were they ever to occur. The mystery shoppers will be instructed to be honest about their sexual behaviors during their visits. By avoiding creating "personas" or "scripts," shoppers will increase the social validity of the assessment and avoid arousing suspicion due to exaggerated or unrealistic scenarios.

The study staff will create and use a secure database to manage the mystery shoppers, site assignments, and testing schedules. Upon completion of the 1-day training, study staff will assign the mystery shoppers a specific day and time for their initial testing site visit. The mystery shoppers will report to the iTech SRV before each scheduled site visit to check in with a staff member and receive their site assignment. They will be loaned a mobile phone equipped with a car share app to use for travel 
to and from testing sites. All car share trips will be tracked and paid for by the study, so no transportation costs will be incurred by the mystery shoppers.

Once at the testing site, the mystery shoppers will state that they have no income, health insurance, or any proof of identification. In doing so, we will be able to ascertain whether these would be potential barriers to testing at a given location and ascertain the lowest possible fees that would be charged to YMSM. As in the original study [34], we will reimburse the mystery shoppers for any charges linked to their testing experiences. Upon completion of a testing visit, the mystery shoppers will use the mobile phone's car share app to travel back to the iTech SRV. They will complete the site assessment survey on the mobile phone or on a computer at the iTech SRV. Mobile phones will be returned to the study staff upon return to the SRV.

The site assessment survey will record shoppers' perceptions of their testing experience, specifically LGBTQ visibility, medical form inclusivity, clinic environment, privacy and confidentiality, PrEP information and dialogue, patient-provider relationship context, patient-provider counseling, safer sex education, perceived provider competency, and participant-provider interactions (Textbox 1). The mystery shoppers will also have the opportunity to leave qualitative feedback in an open text field if they wish to explain any of their responses or record any other information pertinent to their experience that the quantitative assessment did not already capture.

In addition to the site assessment survey, the mystery shoppers will complete a secure video chat session with study staff to discuss their testing experience and have the opportunity to share any adverse interactions. These video chat sessions will not be recorded; their purpose will be to ensure mystery shoppers' safety and prevent subsequent mystery shoppers from being exposed to a site reported to be risky or unsafe (physically or emotionally). Following the video chat session, the mystery shoppers will be given their incentive for the visit and their next visit with study staff will be scheduled before leaving the iTech SRV.

\section{Outcomes}

The mystery shoppers' site assessment scores will be aggregated for use in Phase 2 of the research activities: RCT to test the efficacy of GC. Specifically, the scores for each site will be averaged and embedded in the intervention condition of the GC Web app: when participants search for testing sites they will only see sites that rank in the top 50\% for that city, sorted from highest to lowest ranking.

\section{Phase 2: Randomized Controlled Trial}

\section{Design}

The research activities involve a 2-arm 12-month prospective RCT enrolling $480 \mathrm{HIV}$-negative or status-unaware YMSM (age 15-24 years). After assent or consent and completion of a baseline survey, YMSM will be randomized on a 1:1 basis to either the control or intervention condition (intervention, $n=240$; control, $n=240$ ). Participants randomized to the control condition will be directed to the AIDSVU.org testing site locator. While the provision of a test locator is a low intensity intervention, we felt that withholding referrals to testing and care services would be unethical given YMSM's vulnerability to HIV and STIs. Furthermore, given the availability of search engines to locate HIV or STI testing sites, the test locator condition may be considered usual care. Nevertheless, by providing the existing testing site locator only, we will still be able to test the effect of GC (ie, user-tailored content focused on HIV or STI testing and PrEP referral and the linkage to high-quality agencies). Web-based study assessments are conducted every 3 months across the intervention and control conditions, with a total follow-up period of 12 months. At the end of RCT, we will make the intervention accessible to YMSM in the control condition.

\section{Intervention}

The GC intervention was developed by customizing content based on YMSM's psychosocial and sexual profiles (eg, sociodemographics, HIV and STI testing history and testing motivations, recent sexual behavior, sources of support, self-reported values), as reported by participants' answers to their baseline assessment. At the individual level, GC delivers tailored Web-based content specific to each user's demographic characteristics (eg, age, race or ethnicity, location, relationship status), HIV and STI risk behaviors (eg, HIV and STI testing history, substance use, communication with partners regarding status), and sociocultural context (eg, homelessness, incarceration). GC also employs tailoring at the system level using mystery shopper scores. Participants across both conditions who have been tested will be asked to rate their visit at their quarterly follow-up assessment using the same mystery shopper criteria. Sites will receive biannual summaries, including the aggregated user reviews and brief technical assistance reports, to help sites understand their performance based on quality assurance evaluations from YMSM clients and to optimize service delivery, if needed.

For the participants in the intervention condition, the tailored Web app has 4 sections of content: "What," "Why," "How," and "Where." The "What" section is split into 3 pages: "Facts," "STIs," and "Tests." On each of those pages, topics are displayed in boxes that are randomly organized and open to display additional information if the user clicks or presses. The Facts page (Figure 1) displays boxes that contain general prevention facts (eg, "You won't always know if someone has an STI.") relevant to this population. On the STIs page, if a participant clicks "chlamydia," they receive additional information about how it can be contracted, possible symptoms, testing options, and treatment options (if applicable). The Tests page displays boxes with each HIV or STI testing method (eg, blood test, swab test, urine test), and each box contains more specific information (eg, what STIs it tests for, steps for the test) upon click or press. 
Textbox 1. Clinic and provider interaction visits to be recorded by the mystery shoppers.

\section{Clinic characteristics}

Session speed (min)

LGBT visibility (Cronbach alpha $=0.84$ )

- The clinic has symbols aimed at lesbian, gay, bisexual, transgender (LGBT) people (eg, equal sign, rainbow flag)

- The clinic has printed materials (eg, brochures) aimed at LGBT people

- The clinic has LGBT welcoming symbols

Medical forms (Cronbach alpha $=0.59$ )

- The clinic uses LGBT-inclusive language on medical forms

- $\quad$ The clinic uses transgender-inclusive language on medical forms

Clinic environment (Cronbach alpha $=0.76)$

- The office staff were generally friendly

- The office staff were judgmental (Reverse coded)

- The office staff were not lesbian, gay, bisexual, transgender, questioning or queer (LGBTQ)-sensitive (Reverse coded)

- I felt uncomfortable in the waiting room (Reverse coded)

- $\quad$ The clinic used LGBT-affirming language when speaking to me

Privacy and confidentiality

- The clinic staff kept patient information confidential

- Interactions between clients and staff were kept private

- The provider explained confidentiality (either verbally or via a document)

Pre-exposure prophylaxis (PrEP)-specific indicators

- The clinic had information about PrEP

- The clinic offers PrEP or PrEP referrals

\section{Provider exchanges}

Relationship context (Cronbach alpha $=0.89$ )

- The provider asked me about my sexual orientation

- The provider asked me about my relationship status

- The provider asked if I had experienced intimate partner violence

Counselling session (Cronbach alpha $=0.76$ )

- The provider explored my motivation for testing

- The provider offered to help me set goals

- The provider offered to help me set action steps to meet safer sex goals

- The provider offered me risk reduction options

- The provider's recommendations were valuable

Safer sex education (Cronbach alpha $=0.88$ )

- The provider made sure I knew how to use a condom

- The provider helped me identify a condom that works for me

- The provider helped me identify a lube that works for me

- The provider discussed PrEP as a prevention strategy with me

Perceived provider competency (Cronbach alpha $=0.65$ )

- The provider or test counsellor appeared knowledgeable about HIV and STIs 
- The provider appeared knowledgeable about LGBTQ health issues

Negative provider interactions (Cronbach alpha=0.89)

- $\quad$ The provider made me feel comfortable (Reverse coded)

- I felt pressured by the provider to adopt specific risk reduction options

- The provider was judgmental about the kind of sex I have (eg, anal, receptive, or penetrative, etc)

- The provider was judgmental about how many partners I have had

- The provider was judgmental about how I met my partners

Figure 1. HIV and STIs (sexually transmitted infections) facts.

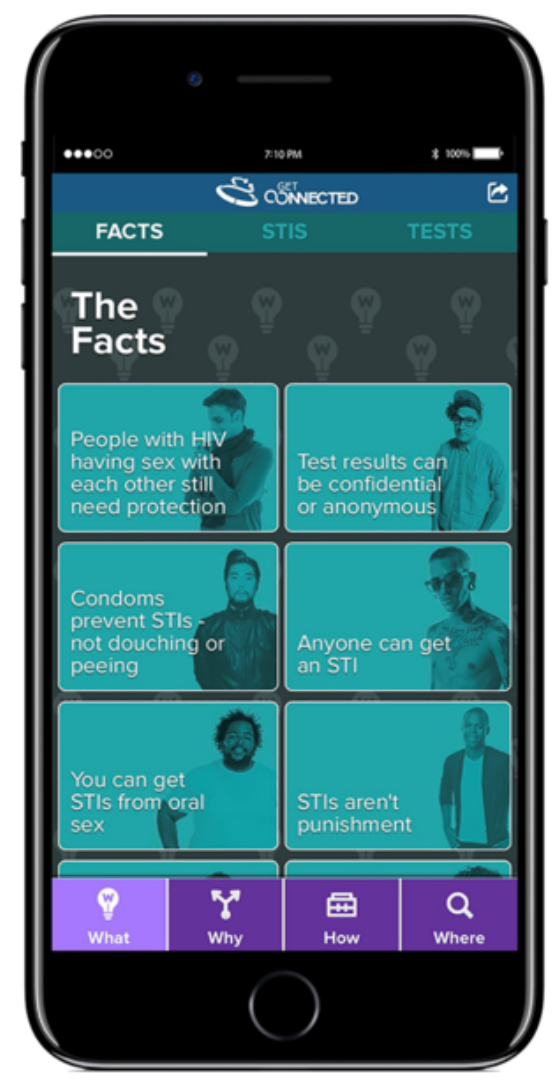

The second section focuses on the "Why" on 2 pages: "Values" and "Pros and Cons." The basic design and functionality is the same as described above in the "What" section. The Values page (Figure 2) encourages participants to assess their motivations, values, and strengths regarding HIV or STI testing. Reasons for getting tested are tailored to participants' testing history (eg, "Never tested" versus "Tested for HIV, but not STIs") in order to acknowledge their prior behaviors. Building on best practices, persuasive messages regarding the importance of linking to prevention services are then presented by linking participants' values from the baseline survey (eg, being attractive, being religious, being sexy, being loved, being athletic, etc) to the desired outcomes. For example, a participant who indicated he valued being religious may see a message that says, "Finding strength in your faith. Your religious beliefs are important to you. Getting tested is one way to take care of, and honor, the body that you've been given. How might you draw on your faith to find strength to get tested?" The Pros and Cons page presents information on the perceived benefits and barriers of getting tested and of not getting tested.

The third section is about the "How" of testing and includes pages on potential "Barriers" to getting tested and "Supports" that may help a participant decide to get tested. Barriers (Figure 3 ) include issues like financial costs, social norms, and prioritization, which may affect participants' desire to get tested for HIV or STIs. "Supports" has information on how their strengths and social support systems can help them make a choice about testing. Recognizing that barriers and supports may shift over time, content on these pages is tailored to identify the most recent barriers and supports as indicated by YMSM in their most recent survey.

The final section is the "Where" of testing and includes a page where a user can "Customize" their search for nearby testing sites (Figure 4) and a "Your Sites" page that displays testing sites based on that customization. Participants can customize their search based on many clinic characteristics, including 
whether walk-in appointments are available, if they have weekend hours, and if they accept insurance. The "Your Sites" page is a listing of providers (including contact and location information) based on the participant's customization selections. Testing sites are initially ranked using an algorithm that accounts for each site's average mystery shopping scores. These scores are updated as participants get tested and rate sites over the 12-month study period. Participants can choose sites they may want to visit and then have the site information emailed or texted to them. Along with any site a participant emails or texts to themselves, they will be provided with 7 questions they can ask a provider during a testing visit. These questions were developed by the GC youth and community advisory boards and were found to be helpful to pilot trial participants when they encountered test counselors who were not perceived to be effective.

\section{Participants}

Eligible participants will be those assigned male sex at birth who currently identify as male, aged 15-24 years (inclusive) at the time of screening, have had consensual anal sex with another man in the past 6 months, self-report as HIV-negative or unsure of their HIV status, have access to a computer or mobile phone, can read and speak English, and live within the city limits of Philadelphia, Atlanta, or Houston.

Figure 2. Values page.

\section{Sample Size}

Our target enrollment across both conditions is 480 participants (intervention, $\mathrm{n}=240$; control, $\mathrm{n}=240$ ). This number allows for $20 \%$ loss to follow-up rate and a final analytic sample of 400 YMSM across the 3 cities. Participants may continue the study even if they miss assessments intermittently over the data collection period. We will compare those who completed different follow-up assessments with those who did not based on key predictors from the baseline assessment to check for possible bias due to missing data and informative censoring. When appropriate, we will use expectation-maximization algorithm-based imputation methods in our analyses $[35,36]$. The primary outcomes for the proposed trial are successful uptake of HIV prevention services (eg, HIV or STI testing) and PrEP awareness and willingness. For HIV testing, we define power as correctly identifying the difference in the proportion of YMSM who engage in HIV testing 2 or more times at least 3 months apart during the 12-month follow-up period ("frequent tester") in our treatment arm (GC) versus our control arm. For STI testing, we define it as receiving at least 1 STI test. For proportions (eg, HIV testing, PrEP awareness), our sample size calculations are based on a 2-sample test of proportions using a 2-sided significance level of 0.05 .

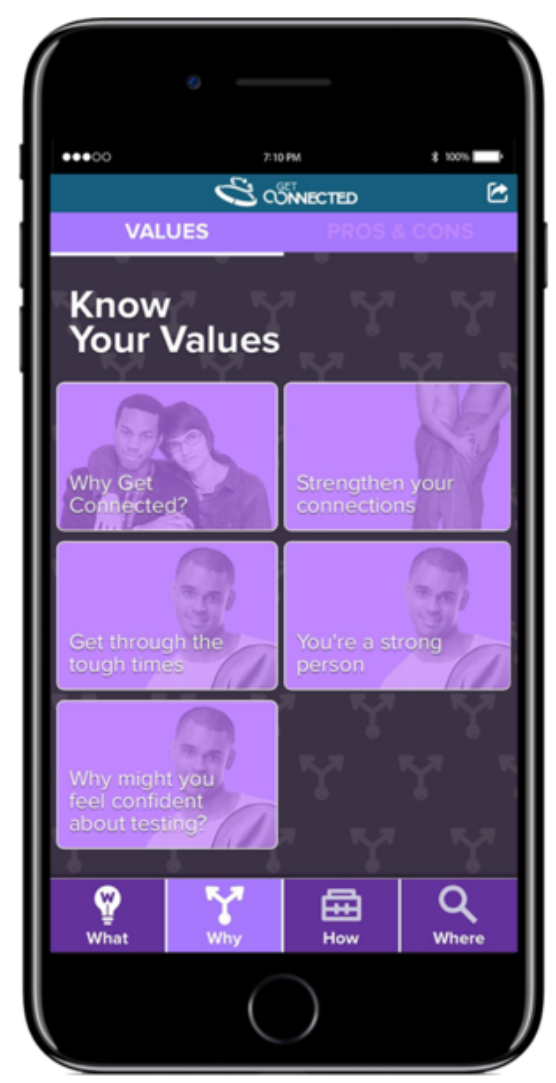


Figure 3. Barriers page.

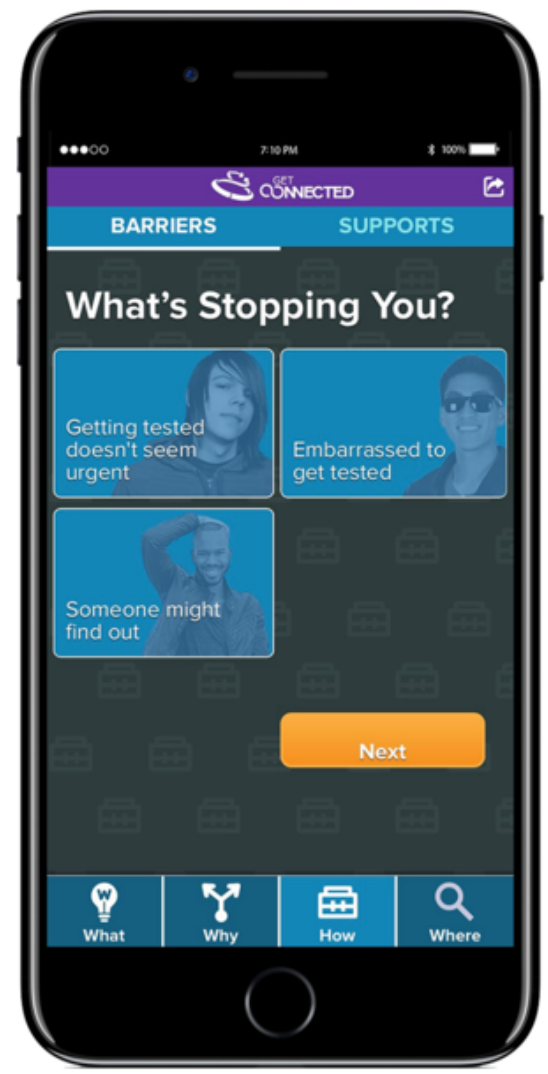

Figure 4. Customization page.

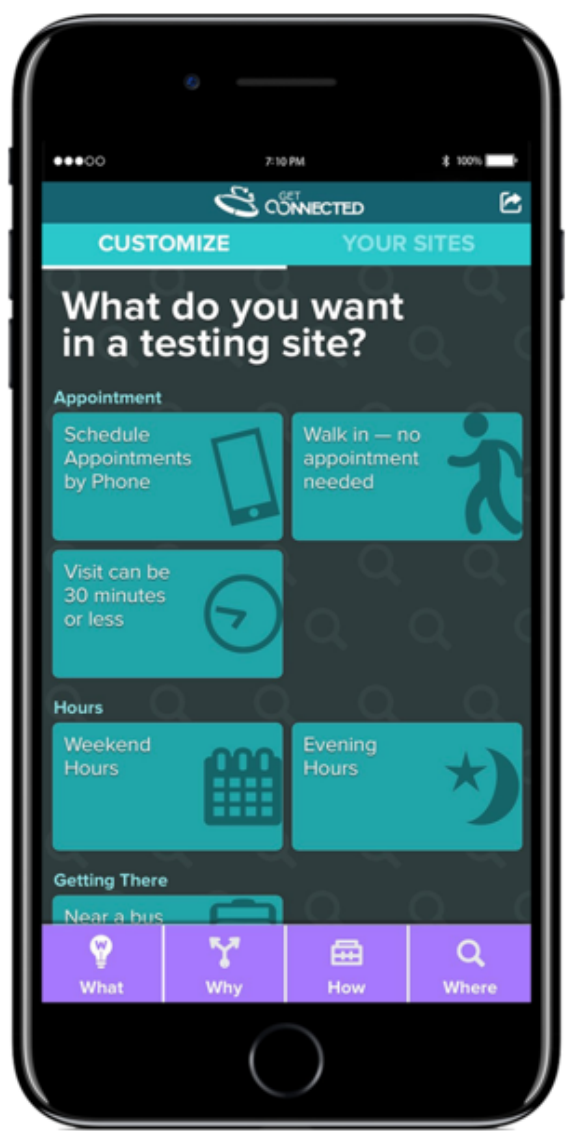


In order to have $80 \%$ power to the intervention and control groups, we require at least 400 participants to find an absolute difference of $13 \%$ in cross-sectional analyses. Assuming a within-person correlation of 0.25 , we can detect an $8.8 \%$ difference, indicating we have power to detect the smallest possible difference between arms. A less favorable within-person correlation of 0.75 allows us to detect an $11.3 \%$ difference. For mean differences across continuous outcomes (eg, PrEP willingness), our sample size calculations are based on a 2 -sample $t$ test, assuming equal variance using a 2 -sided significance level of 0.05 . We are able to detect a between-arm effect size difference of Cohen $d=0.25$ at the final follow-up time point at $80 \%$ power. For repeated measures analyses, assuming a within-person correlation of 0.25 , we would be able to detect an effect size of 0.08 . A less favorable within-person correlation of 0.75 allows us to detect an effect size of 0.11 .

\section{Incentives}

Participants can earn up to US $\$ 155$ total: Baseline survey=US $\$ 20$, month 1 survey=US $\$ 20$, month 3 survey=US $\$ 25$, month 6 survey=US \$30, month 9 survey=US \$30, and month 12 survey=US $\$ 30$.

\section{Randomization}

After assent or consent and completion of the baseline survey, YMSM will be randomized by city 1:1 to either the control or intervention condition (intervention, $\mathrm{n}=240$; control, $\mathrm{n}=240$ ) [37]. The stratified randomization process occurs upon completion of the baseline survey.

\section{Outcomes}

\section{Primary Outcomes}

The primary outcomes relate to the successful uptake of HIV prevention services among our sample of self-reported HIV-negative or serostatus-unaware YMSM. We have considered 3 prevention outcomes: HIV testing, STI testing, and PrEP awareness and willingness.

\section{HIV Testing}

The baseline survey will include questions on lifetime HIV testing history. Follow-up surveys will repeat the questions from the baseline and will also include questions on HIV testing in the prior 3-month period, including test results. The HIV testing outcome will be the proportion of YMSM tested for HIV 2 or more times at least 3 months apart in the 12-month follow-up period ("frequent tester"). As an additional analysis, we will also examine the proportions of participants who receive $1 \mathrm{HIV}$ test.

\section{Sexually Transmitted Infection Testing}

The baseline survey will include separate questions on lifetime testing history of gonorrhea, chlamydia, and syphilis, respectively, as well as questions about ever having a genital exam, an anal pap smear, or a vaccination for Hepatitis A and $\mathrm{B}$, human papilloma virus, and meningitis. Follow-up surveys will repeat the questions from the baseline but will ask about STI testing behavior in the prior 3-month period, including test results if a participant indicates they received a test. The STI testing outcome will be the proportion of YMSM tested for any
STI 2 or more times, at least 3 months apart, in the 12-month follow-up period ("frequent tester"). As an additional analysis, we will also examine the proportions of participants who receive 1 STI test.

\section{Pre-Exposure Prophylaxis Awareness and Willingness}

The survey will contain a brief description of PrEP to orient the participant. Most questions were adapted from recent studies of PrEP attitudes with YMSM [38-41]. PrEP awareness will be a single-item measure of whether the participant has heard of PrEP [39]. For participants who do not report current PrEP use, PrEP willingness will be assessed by asking how likely the participant would be to start PrEP in the next 3 months and the reason(s) why the participant is not currently taking PrEP (eg, never heard of PrEP, worried about side effects, lack of support from friends or family).

\section{Secondary Outcomes}

As secondary outcomes, we will examine the uptake of PrEP, changes in sexual risk behavior, and the linkage and retention in care among newly diagnosed HIV-positive cases. While we expect a small number of newly diagnosed HIV infections, we will measure initiation of antiretroviral therapy and self-reported adherence as a secondary outcome. We are not powered to measure differences in engagement in HIV care across trial arms, so we include this as an exploratory analysis.

\section{Mechanisms of Change}

Consistent with our theoretical framework, we will assess YMSM's psychosocial correlates predicting adoption of HIV services (ie, attitudes, norms, self-efficacy, and behavioral intentions to get HIV tested). Integrated Behavioral Model constructs will be assessed with subscales assessing YMSM's attitudes, social norms, and behavioral intentions [42] that we have used in the past with this population [43]. Social norms assess the extent to which participants feel that friends and family believed the participants should test for HIV. Behavioral intention items assess participants' intention to adopt HIV testing. Self-efficacy to access HIV or STI services and to discuss sexuality-related issues with partners and provider will be ascertained.

\section{Uptake of Pre-Exposure Prophylaxis}

At each follow-up assessment, PrEP-eligible (per CDC guidelines), HIV-negative YMSM will be asked whether they have begun using PrEP [39]. YMSM who report using PrEP will be asked to report their adherence to PrEP.

\section{Sexual Risk Behavior}

Sexual risk behavior will be assessed using the Sexual Practices Assessment Schedule used in previous Web-based studies with YMSM $[44,45]$. This assessment will explore the number of occasions of different sexual acts (oral, anal; receptive, insertive) with 3 different types of partners (romantic interest, casual partner "hookup," or friend with benefits), use of condoms during the past 3 months, and knowledge about partners' HIV status and PrEP use. Assessments ascertain sexual behaviors with male partners and will be conducted at baseline and each follow-up. At-risk sex will be defined as any anal intercourse without condoms or PrEP with a person of known positive and 
detectable viral load or a person of unknown serostatus during the follow-up period. We will assess the number of partners with whom participants had "at-risk sex," as well as estimate the incidence of at-risk sex acts (ie, incidence density: the numerator being number of at-risk sex acts and the denominator being person-years of follow time).

\section{Linkage and Retention in Care Among Newly Diagnosed HIV-Positive Cases}

Among newly diagnosed HIV-positive cases, we will measure participants' linkage and engagement with appropriate medical care after initial diagnosis, using criteria employed in prior ATN protocols with youth [46-49]. We will define linkage as an HIV-related medical visit within 45 days of referral and engagement as a second HIV-related medical visit within 16 weeks of initial visit [48]. Onset of antiretroviral therapy initiation, self-reported adherence to ART, and viral suppression are exploratory indicators [47], as we recognize that our follow-up period may not be a sufficient amount of time to see these changes.

\section{Covariates}

We will also measure the following constructs as potential predictors or moderators in our analyses.

\section{Sociodemographic Information}

We will include questions on participants' race or ethnicity, educational attainment, employment status, place of birth, housing status, and history of incarceration, sexual identity, and "outness" to their social network.

\section{Site Evaluations}

Across both trial arms, YMSM who report testing in the prior 3 months will complete site assessments of their testing experiences to measure comfort, quality, and concerns after visiting a site for HIV or STI testing. The site assessment form is the same form used by the mystery shoppers. We will use these assessments to send aggregate data of YMSM's satisfaction with services to agencies biannually.

\section{Substance Use and Psychological Distress}

Previous studies have demonstrated higher vulnerability to HIV risk behaviors and engagement in prevention and care among YMSM who report alcohol, tobacco, and other drug (ATOD) use and psychological distress; therefore, we will measure both ATOD and psychological distress as potential effect moderators.

We will assess the frequency of ATOD use (as measured in the National Survey on Drug Use and Health) over the past 3 months in the baseline survey and follow-up surveys for alcohol, tobacco products, marijuana, nonprescription drugs, cocaine, amphetamines, inhalants, opioids (including heroin), hallucinogens, and depressants [50]. If respondents indicate any ATOD use within the past 3 months, we will ask, for each substance, how often the substance was used and if it was used immediately before or during sex.

We will measure psychological distress using existing, well-validated scales: the Patient Health Questionnaire-8 (PHQ-8) [51] and the 7-item Generalized Anxiety Disorder (GAD-7) [52] scale. We will use the first 2 items from each scale to screen participants for symptoms of depression and anxiety (PHQ-2 [53] and GAD-2 [54]). Participants who report depressive symptoms (score of 3 or more on PHQ-2) will be asked the last 6 items from PHQ-8. Participants who report symptoms of anxiety (score of 3 or more on GAD-2) will be asked the last 5 items from GAD-7.

\section{Intervention Acceptability}

At each follow-up, participants will report on the acceptability of their assigned study arm. We will use the Systems Usability Scale [55] to ascertain participants' overall satisfaction with the intervention, perception of the information quality, and perceived usefulness of their intervention to improve their health.

\section{Use of Intervention Over Time}

We will measure intervention exposure using paradata from the intervention, including counts of user sessions, length of sessions, pages visited, and functions utilized. This information will assist in examining whether intervention dosage influences the overall efficacy of the intervention and in informing the cost analysis and wider implementation and scalability [56].

\section{Technology and Social Media}

We will include Pew Internet survey questions [57] regarding the use of different devices, the number of hours spent online through each device, reasons for social media use, sites commonly frequented, and extent to which the internet supplements face-to-face interactions. We will also measure participants' frequency of social media use to look for HIV or sexual health-related information $[43,58]$ and their online partner-seeking behaviors $[59,60]$. We will ask these questions at each follow-up, except for the 1-month follow-up. We will also use the eHealth Literacy Scale [61] to assess participants' perceived ability to use the internet to find health resources.

\section{Statistical Analysis}

Descriptive statistics of the psychosocial and demographic characteristics of the participants will be used to describe all participants. These will be compared between treatment groups using $t$ tests or Wilcoxon rank sum tests for continuous variables and chi-square tests for categorical variables. To test for intervention efficacy, we will conduct primary analyses of our primary outcomes (HIV testing, STI testing, and PrEP awareness and willingness) using regression analyses to compare our treatment and control groups using the appropriate link function (identity for continuous outcome, logit for binary outcome, and natural $\log$ for count outcomes). Interactions between group assignment and these characteristics will be tested to explore the potential moderators of treatment effect. We will repeat these analyses for the secondary outcomes (eg, theoretical mediators, sexual risk behaviors, sexual risk behaviors, PrEP uptake).

We will use the general framework of generalized linear mixed models (GLMM) to test for intervention effects over time. Note that some of our outcomes are binary, some are count, and some continuous traits and thus need to be treated differently. The general form of GLMM will be $\mathrm{g}\left(\mu_{i j}\right)=\beta_{0 i}+\beta_{\text {cov }}$ Covariates $_{i j}$ $+\beta_{\text {Time }}$ Times $_{j}+\beta_{\text {Arm } \times \text { Time }}$, where $\mu_{i j}$ is the mean response 
corresponding to subject $i$ at Time $j$ (baseline and 4 follow-ups), with its appropriate link function (identity for continuous outcome, logit for binary outcome, and natural log for count outcomes); $\operatorname{Tr} t_{\mathrm{i}}=1$ if the $\mathrm{i}^{\text {th }}$ subject is in the intervention group, and $T r t_{\mathrm{i}}=0$ if the $\mathrm{i}^{\text {th }}$ subject is in the control group. The interaction coefficients $\beta_{\text {Trt } \times \text { Time }}$ are of interest here, measuring the difference in the rate of change in outcome across the 2 treatment groups over time. The subject-specific random intercepts $\beta_{0 i}$ are assumed to be normally distributed with a common variance and they account for within-person correlation. We will also explore if we need a subject-specific random slope corresponding to visit in the above model. Maximum likelihood estimation will be used for fixed effect parameters.

Models will be compared according to the information criteria such as Akaike Information Criterion and Bayesian Information Criterion. For some binary outcomes, such as HIV testing, we will perform an aggregate analysis after collapsing across the repeated measures using simple logistic regression, comparing whether the probability of having tested at least once over the entire follow-up period is different across treatment groups, after adjusting for baseline values. To ensure robustness, we will also apply an exchangeable working correlation structure to its corresponding generalized estimating equation model. We will conduct exploratory regression analyses to examine regional differences. These regressions will be run with group assignment and region in the model, controlling for sociodemographic characteristics. Interactions between group assignment and region will be tested to explore potential site-specific moderators of treatment effect.

As a secondary analysis, we will build on our GLMM framework to examine whether the intervention effects in the theoretical mediators (eg, attitudes, norms, and self-efficacy) are associated with our outcomes. We will also test whether these relationships vary as a function of YMSM's varying engagement with the intervention (intervention acceptability, use of intervention over time). Interactions between group assignment and these characteristics will test for potential moderators of treatment effect.

\section{Cost Analysis}

In order to inform the eventual scale-up of GC, we will also conduct a cost analysis of GC and control conditions to inform discussions of sustainability and roll out of the GC intervention. We will collect information on costs associated with the delivery of the intervention. No costs associated with research data collection will be included. These components of cost will be summed over the 12-month study period for each participant to generate an estimated per person cost. Effectiveness will be measured by examining HIV-related outcomes reported by YMSM over the 12-month period. Incremental cost effectiveness ratio (ICER) across treatment arms will be defined as delta $\mathrm{C}$ or delta $\mathrm{E}$, where delta $\mathrm{C}$ denotes the estimated difference in mean cost of the intervention and delta $E$ reflects the estimated difference in mean effectiveness between the intervention and control groups. Nonparametric bootstrap resampling will be used to estimate the $95 \%$ CI of incremental cost effectiveness ratio [62]. Analysis will be performed on participants with complete data. Sensitivity analysis will be conducted by including all participants with multiple imputations for those with missing data.

\section{Qualitative Assessment of Testing Sites' Satisfaction}

We will qualitatively assess testing sites' satisfaction with the biannual performance assessments and their improvements in service delivery when working with YMSM across the 3 regions. Ten site directors will be randomly selected from testing sites in each city. Eligible participants will be able to read and speak English and serve as the site director of an HIV or STI testing site in Philadelphia, Atlanta, or Houston. We will conduct semistructured qualitative in-depth interviews (60-90 minutes) that focus on 4 domains: (1) existing prevention services used and promoted by the agency, (2) agency (internal) resources currently missing, that if identified and addressed, could improve the delivery of HIV, STI, and PrEP services to YMSM, (3) feedback on the biannual performance assessments and their use for service delivery improvements, and (4) the advantages and disadvantages of GC rollout within AIDS Service Organizations.

Interviews will occur via teleconference to maximize candidness and privacy while decreasing travel-related costs. We will use VSee, a simple and low-cost video chat platform that requires no server infrastructure to set up or maintain and allows providers to be HIPAA-compliant. Interviews will be audio-recorded to allow for verbatim transcription, and then checked for accuracy and completion. Initial reading and coding of the transcripts will be reviewed, compared, and refined in team meetings. This systematic process will lead to the creation of a coding structure that includes a hierarchical set of constructs seen in the data. We will analyze several transcripts jointly to establish intercoder reliability. The team will then code all transcripts using our coding structure and add inductive codes during the iterative analysis process. Throughout, we will discuss emerging themes, resolve difficulties or concerns that may arise, and adapt the codebook as necessary.

Since we seek to gain a multilevel understanding of the structural, organizational, and interpersonal barriers and facilitators of implementing GC, our analysis will utilize a phenomenological framework [63]. Although our analysis will rely primarily on a phenomenological inductive approach, we will also employ aspects of deductive analysis that consider our guiding conceptual framework. This combination of analytic strategies will enable us to conduct a phenomenological analysis (inductive) that was initially informed by existing research and theory via the conceptual framework (deductive). We will analyze the qualitative data using thematic analysis until we have reached saturation [64-66].

\section{Results}

GC research activities began in September 2016 and are ongoing. Institutional review board (IRB) submission is complete, with IRB authorization agreements being finalized across the participating universities and SRVs. 


\section{Discussion}

There are several potential challenges and limitations to the proposed clinical trial. First, we will rely on self-reported outcomes. We will not include biological measures (eg, presence of HIV or STI), as we would have to dramatically increase our sample size to detect significant effects in biomarkers among newly diagnosed HIV or STI cases and it would be inefficient to collect biomarkers in a Web-based study. We will frame the presentation of results as self-reported outcomes. Second, we propose to recruit a diverse (in terms of race or ethnicity and age) sample of 15-24 year-old participants. It is possible that we may experience more success in recruiting older YMSM (those aged $>18$ years). To counteract this, we will include a broad range of social media outlets in our recruitment, allowing us the potential to recruit our full age range. Collectively, the team has a vast experience of recruiting youth into HIV research efforts and substantial experience in recruiting online samples of urban race or ethnic minority YMSM. Third, we are unable to untangle race from Latino ethnicity, as it would require a much larger sample size to examine race by ethnicity subgroup differences. Because we propose to quota sample across race or ethnicity in each of the regions, the breakdown of Latino race would create some small sample sizes. Fourth, we recognize that socioeconomically disadvantaged participants may require access to a computer or secure Wi-Fi connection to participate fully in the study. YMSM who are interested in participating but require access or who prefer to complete assessments at a study location will be able to complete intervention activities at their local iTech SRV. Finally, to minimize potential risks, all iTech SRVs have specific policies governing the treatment of human participants, including the referral to medical and psychological services in the event a participant should report a need for these services or experience any adverse reactions resulting from study procedures.

With increasingly promising evidence of the efficacy of biomedical prevention tools, such as PrEP, for reducing the risk of HIV infection among MSM [38,39,67-70], there is increased attention to the potential for HIV testing to act as a gateway to other HIV prevention tools and care efforts [71,72]. Many of the cognitive and behavioral risk factors that contribute to the high rates of HIV infection among MSM are established during adolescence and the transition into young adulthood. This age should be considered a priority time for intervening on cognitive and behavioral risks for HIV, while also introducing YMSM to HIV testing as a gateway to other HIV prevention options.

Efforts to encourage and motivate YMSM to engage in repeat HIV or STI testing or to adopt other prevention efforts (eg, PrEP $[38,39,67])$ may be diminished if structural barriers (eg, medical mistrust, lack of insurance or transportation) and cultural insensitivity to YMSM's needs (eg, racial or ethnic and sexual orientation stigma) lead to delays or avoidance of HIV or STI services $[10,73,74]$. HIV prevention tools must be designed to help YMSM overcome a series of multilevel barriers at the individual (eg, risk awareness), systems (eg, costs, lack of culturally competent care), and structural (eg, homelessness, stigma) levels. Developing strategies to promote the use of HIV prevention services among YMSM requires the creation of interventions such as GC that are culturally sensitive to their psychosocial needs [13] and facilitate access to comprehensive sexual health services [14]. If proven efficacious, GC has the potential to fill a gap in HIV prevention by providing a Web-based, tailored intervention that allows YMSM to learn about local prevention services and to build the skills necessary for successful adoption of prevention.

\section{Acknowledgments}

This work was supported by the National Institutes of Health Adolescent Medicine Trials Network for HIV/AIDS Interventions (ATN 139; MPI: Bauermeister \& Stephenson) as part of and the UNC/Emory Center for Innovative Technology (iTech; PIs: Drs Hightow-Weidman/Sullivan, 1U19HD089881). The content is solely the responsibility of the authors and does not represent the official views of the funding agencies. The authors would like to thank Adi Ferrara, MS, ELS, for help in the preparation of this manuscript.

\section{Conflicts of Interest}

None declared.

\section{References}

1. Grossman CI, Forsyth A, Purcell DW, Allison S, Toledo C, Gordon CM. Advancing novel HIV prevention intervention research with MSM--meeting report. Public Health Rep 2011;126(4):472-479 [FREE Full text] [doi: 10.1177/003335491112600404] [Medline: 21800742]

2. CDC. National Center for HIV/AIDS, Viral Hepatitis, STD, and TB Prevention. 2014. HIV and Young Men Who Have Sex With Men URL: https://www.cdc.gov/healthyyouth/sexualbehaviors/pdf/hiv factsheet ymsm.pdf [accessed 2018-07-10] [WebCite Cache ID 70nukGSUc]

3. Hall HI, Holtgrave DR, Tang T, Rhodes P. HIV transmission in the United States: considerations of viral load, risk behavior, and health disparities. AIDS Behav 2013 Jun;17(5):1632-1636. [doi: 10.1007/s10461-013-0426-z] [Medline: 23456577]

4. Office of National AIDS Policy. Office of National AIDS Policy. Washington, D.C: The White House; 2010. The National HIV/AIDS Strategy for the United States URL: https://obamawhitehouse.archives.gov/sites/default/files/uploads/NHAS. pdf [accessed 2018-07-10] [WebCite Cache ID 70nvK7t3a] 
5. Krueger A, Dietz P, Van HM, Belcher L, Johnson AS. Estimates of CDC-Funded and National HIV Diagnoses: A Comparison by Demographic and HIV-related Factors. AIDS Behav 2016 Dec;20(12):2961-2965. [doi: 10.1007/s10461-016-1293-1] [Medline: 26796383]

6. Bauermeister JA, Pingel ES, Jadwin-Cakmak L, Meanley S, Alapati D, Moore M, et al. The use of mystery shopping for quality assurance evaluations of HIV/STI testing sites offering services to young gay and bisexual men. AIDS Behav 2015 Oct;19(10):1919-1927 [FREE Full text] [doi: 10.1007/s10461-015-1174-z] [Medline: 26303197]

7. Harper GW, Riplinger AJ. HIV prevention interventions for adolescents and young adults: what about the needs of gay and bisexual males? AIDS Behav 2013 Mar;17(3):1082-1095. [doi: 10.1007/s10461-012-0178-1] [Medline: 22460226]

8. Harper G, Schneider M. Oppression and Discrimination among Lesbian, Gay, Bisexual, and Transgendered People and Communities: A Challenge for Community Psychology. American journal of community psychology 2003;31(3):A. [doi: 10.1023/A:1023906620085]

9. Hosek SG, Harper GW, Lemos D, Martinez J. An Ecological Model of Stressors Experienced by Youth Newly Diagnosed With HIV. J HIV AIDS Prev Child Youth 2008 Jul;9(2):192-218 [FREE Full text] [doi: 10.1080/15538340902824118] [Medline: 20216916]

10. Hussen SA, Harper GW, Bauermeister JA, Hightow-Weidman LB, Adolescent Medicine Trials Network for HIV/AIDS Interventions. Psychosocial influences on engagement in care among HIV-positive young black gay/bisexual and other men who have sex with men. AIDS Patient Care STDS 2015 Feb;29(2):77-85 [FREE Full text] [doi: 10.1089/apc.2014.0117] [Medline: 25682888]

11. Ziff MA, Harper GW, Chutuape KS, Deeds BG, Futterman D, Francisco VT, Adolescent Medicine Trials Network for HIV/AIDS Intervention. Laying the foundation for Connect to Protect: a multi-site community mobilization intervention to reduce HIV/AIDS incidence and prevalence among urban youth. J Urban Health 2006 May;83(3):506-522 [FREE Full text] [doi: 10.1007/s11524-006-9036-7] [Medline: 16739051]

12. Ziff MA, Willard N, Harper GW, Bangi AK, Johnson J, Ellen JM. Connect to Protect Researcher-Community Partnerships: Assessing Change in Successful Collaboration Factors over Time. Glob J Community Psychol Pract 2010 Jan;1(1):32-39 [FREE Full text] [Medline: 21152354]

13. Harper GW. Sex isn't that simple: culture and context in HIV prevention interventions for gay and bisexual male adolescents. Am Psychol 2007 Nov;62(8):803-819. [doi: 10.1037/0003-066X.62.8.806] [Medline: 18020756]

14. Sumartojo E, Lyles C, Choi K, Clark L, Collins C, Grey CG, City Study Team. Prevalence and correlates of HIV testing in a multi-site sample of young men who have sex with men. AIDS Care 2008 Jan;20(1):1-14. [doi: 10.1080/09540120701450425] [Medline: 18278609 ]

15. Harper GW, Willard N, Ellen JM, Adolescent Medicine Trials Network for HIV/AIDS Interventions. Connect to Protect ${ }^{\circ}$ utilizing community mobilization and structural change to prevent HIV infection among youth. J Prev Interv Community 2012 Apr;40(2):81-86 [FREE Full text] [doi: 10.1080/10852352.2012.660119] [Medline: 24188350]

16. Garofalo R, Harper GW. Not all adolescents are the same: addressing the unique needs of gay and bisexual male youth. Adolesc Med 2003 Oct;14(3):595-611, vi. [doi: 10.1016/S1041349903500470] [Medline: 15122163]

17. Mustanski BS, Newcomb ME, Du Bois SN, Garcia SC, Grov C. HIV in Young Men Who Have Sex with Men: A Review of Epidemiology, Risk and Protective Factors, and Interventions. Journal of Sex Research 2011 Feb 28;48(2-3):218-253. [doi: $10.1080 / 00224499.2011 .558645]$

18. Cozier N, Gomez M. Geo-location, collaboration, MHealth \& More: Navigating HIV prevention and services across the federal government with one simple tool. 2011 Presented at: National Conference on Health Communication, Marketing, and Media; 8/9-11/2011; Atlanta, GA URL: https://cdc.confex.com/cdc/nphic11/webprogram/Paper27063.html

19. Beardsell S, Coyle A. A review of research on the nature and quality of HIV testing services: A proposal for process-based studies. Social Science \& Medicine 1996 Mar;42(5):733-743. [doi: 10.1016/0277-9536(95)00145-X]

20. Worthington C, Myers T. Desired elements of HIV testing services: test recipient perspectives. AIDS Patient Care STDS 2002 Nov;16(11):537-548. [doi: 10.1089/108729102761041092] [Medline: 12513902]

21. Calabrese SK, Earnshaw VA, Underhill K, Hansen NB, Dovidio JF. The impact of patient race on clinical decisions related to prescribing HIV pre-exposure prophylaxis (PrEP): assumptions about sexual risk compensation and implications for access. AIDS Behav 2014 Feb;18(2):226-240 [FREE Full text] [doi: 10.1007/s10461-013-0675-x] [Medline: 24366572]

22. Knight RE, Shoveller JA, Carson AM, Contreras-Whitney JG. Examining clinicians' experiences providing sexual health services for LGBTQ youth: considering social and structural determinants of health in clinical practice. Health Educ Res 2014 Aug;29(4):662-670 [FREE Full text] [doi: 10.1093/her/cyt116] [Medline: 24412811]

23. Tanner AE, Philbin MM, Duval A, Ellen J, Kapogiannis B, Fortenberry JD, Adolescent Trials Network for HIV/AIDS Interventions. "Youth friendly" clinics: considerations for linking and engaging HIV-infected adolescents into care. AIDS Care 2014 Feb;26(2):199-205 [FREE Full text] [doi: 10.1080/09540121.2013.808800] [Medline: 23782040]

24. Dittus PJ, De RCJ, Jeffries RA, Afifi AA, Cumberland WG, Chung EQ, et al. The project connect health systems intervention: linking sexually experienced youth to sexual and reproductive health care. J Adolesc Health 2014 Oct;55(4):528-534. [doi: 10.1016/j.jadohealth.2014.04.005] [Medline: 24856358] 
25. Hightow-Weidman LB, Muessig KE, Bauermeister J, Zhang C, LeGrand S. Youth, Technology, and HIV: Recent Advances and Future Directions. Curr HIV/AIDS Rep 2015 Dec;12(4):500-515. [doi: 10.1007/s11904-015-0280-x] [Medline: 26385582]

26. Armitage CJ, Conner M. Social cognition models and health behaviour: A structured review. Psychology \& Health 2000 Mar;15(2):173-189. [doi: 10.1080/08870440008400299]

27. Hornik R. An extension of the theory of reasoned actionits successors to multiple behavior interventions. In: Prediction and Change of Health Behavior: Applying the Reasoned Action Approach. Mahwah, NJ: Lawrence Erlbaum Associates; 2007:53-68.

28. Deci EL, Eghrari H, Patrick BC, Leone DR. Facilitating Internalization: The Self-Determination Theory Perspective. J Personality 1994 Mar;62(1):119-142. [doi: 10.1111/j.1467-6494.1994.tb00797.x]

29. Ryan RM, Deci EL. Self-determination theory and the facilitation of intrinsic motivation, social development, and well-being. American Psychologist 2000;55(1):68-78. [doi: 10.1037/0003-066X.55.1.68]

30. Resnicow K, Braithwaite R, Dilorio C, Glanz K. Applying Theory to Culturally Diverse Unique Populations. In: Glanz K, Rimer BK, Lewis FM, editors. Health Behavior and Health Education. Hoboken, New Jersey, United States 3rd ed: John Wiley \& Sons, Inc; 2002:485-509.

31. Resnicow K, DiIorio C, Soet JE, Borrelli B, Hecht J, Ernst D. Motivational interviewing in health promotion: It sounds like something is changing. Health Psychology 2002;21(5):444-451. [doi: 10.1037//0278-6133.21.5.444]

32. Naar-King S, Parsons JT, Johnson AM. Motivational interviewing targeting risk reduction for people with HIV: a systematic review. Curr HIV/AIDS Rep 2012 Dec;9(4):335-343. [doi: 10.1007/s11904-012-0132-x] [Medline: 22890780]

33. Miller W, Rollnick S. Motivational interviewing: helping people change. In: Motivational interviewing: helping people change. 3rd ed. Portland: Ringgold Inc; 2012.

34. Bauermeister JA, Pingel ES, Jadwin-Cakmak L, Harper GW, Horvath K, Weiss G, et al. Acceptability and Preliminary Efficacy of a Tailored Online HIV/STI Testing Intervention for Young Men who have Sex with Men: The Get Connected! Program. AIDS Behav 2015 Feb 1;19(10):1860-1874. [doi: 10.1007/s10461-015-1009-y]

35. Raghunathan TE. What do we do with missing data? Some options for analysis of incomplete data. Annu Rev Public Health 2004 Apr;25:99-117. [doi: 10.1146/annurev.publhealth.25.102802.124410] [Medline: 15015914]

36. Youk AO, Stone RA, Marsh GM. A method for imputing missing data in longitudinal studies. Ann Epidemiol 2004 May;14(5):354-361. [doi: 10.1016/j.annepidem.2003.09.010] [Medline: 15177275]

37. Kernan W, Viscoli C, Makuch R, Brass L, Horwitz R. Stratified randomization for clinical trials. J Clin Epidemiol 1999 Jan;52(1):19-26. [Medline: 9973070]

38. Eaton LA, Driffin DD, Bauermeister J, Smith H, Conway-Washington C. Minimal Awareness and Stalled Uptake of Pre-Exposure Prophylaxis (PrEP) Among at Risk, HIV-Negative, Black Men Who Have Sex with Men. AIDS Patient Care STDS 2015 Aug;29(8):423-429 [FREE Full text] [doi: 10.1089/apc.2014.0303] [Medline: 26083143]

39. Bauermeister J, Meanley S, Pingel E, Soler J, Harper G. PrEP awareness and perceived barriers among single young men who have sex with men. Curr HIV Res 2013 Oct;11(7):520-527 [FREE Full text] [Medline: 24476355]

40. Sullivan P, Sineath C, Kahle E, Sanchez T. Sullivan P, Sineath C, Kahle E, Sanchez T, editors. Awareness, willingness and use of oral PrEP among a national sample of US men who have sex with men, 2013-2015. 2015 Presented at: AIDSImpact; 7/28-31/2015; Amsterdam, Netherlands.

41. Mustanski B, Johnson AK, Garofalo R, Ryan D, Birkett M. Perceived likelihood of using HIV pre-exposure prophylaxis medications among young men who have sex with men. AIDS Behav 2013 Jul;17(6):2173-2179 [FREE Full text] [doi: 10.1007/s10461-012-0359-y] [Medline: 23128980]

42. Fisher CM. Are Information, Motivation, and Behavioral Skills Linked with HIV-Related Sexual Risk among Young Men Who Have Sex with Men? J HIV AIDS Soc Serv 2011;10(1):5-21 [FREE Full text] [doi: 10.1080/15381501.2011.549064] [Medline: 21731473]

43. Meadowbrooke CC, Veinot TC, Loveluck J, Hickok A, Bauermeister JA. Information behavior and HIV testing intentions among young men at risk for HIV/AIDS. J Assn Inf Sci Tec 2014 Jan 15;65(3):609-620. [doi: 10.1002/asi.23001]

44. Carballo-Diéguez A, Dolezal C, Ventuneac A. Sexual Practices Assessment Schedule. New York: HIV Center for Clinical and Behavioral Studies, Columbia University \& New York State Psychiatric Institute; 2002.

45. Bauermeister JA. Sexual Partner Typologies Among Single Young Men Who Have Sex with Men. AIDS Behav 2014 Oct 31;19(6):1116-1128. [doi: 10.1007/s10461-014-0932-7]

46. Hussen SA, Harper GW, Bauermeister JA, Hightow-Weidman LB, Adolescent Medicine Trials Network for HIV/AIDS Interventions. Psychosocial influences on engagement in care among HIV-positive young black gay/bisexual and other men who have sex with men. AIDS Patient Care STDS 2015 Feb;29(2):77-85 [FREE Full text] [doi: 10.1089/apc.2014.0117] [Medline: 25682888]

47. Kahana SY, Fernandez MI, Wilson PA, Bauermeister JA, Lee S, Wilson CM, et al. Rates and correlates of antiretroviral therapy use and virologic suppression among perinatally and behaviorally HIV-infected youth linked to care in the United States. J Acquir Immune Defic Syndr 2015 Feb 01;68(2):169-177 [FREE Full text] [doi: 10.1097/QAI.0000000000000408] [Medline: 25590270] 
48. Philbin MM, Tanner AE, DuVal A, Ellen JM, Xu J, Kapogiannis B, Adolescent Trials Network for HIV/AIDS Interventions. Factors affecting linkage to care and engagement in care for newly diagnosed HIV-positive adolescents within fifteen adolescent medicine clinics in the United States. AIDS Behav 2014 Aug;18(8):1501-1510 [FREE Full text] [doi: 10.1007/s10461-013-0650-6] [Medline: 24682848]

49. Fortenberry JD, Martinez J, Rudy BJ, Monte D, Adolescent Trials Network for HIV/AIDS Interventions. Linkage to care for HIV-positive adolescents: a multisite study of the adolescent medicine trials units of the adolescent trials network. J Adolesc Health 2012 Dec;51(6):551-556 [FREE Full text] [doi: 10.1016/j.jadohealth.2012.03.012] [Medline: 23174464]

50. Bauermeister JA, Zimmerman MA, Johns MM, Glowacki P, Stoddard S, Volz E. Innovative Recruitment Using Online Networks: Lessons Learned From an Online Study of Alcohol and Other Drug Use Utilizing a Web-Based, Respondent-Driven Sampling (webRDS) Strategy. J. Stud. Alcohol Drugs 2012 Sep;73(5):834-838. [doi: 10.15288/jsad.2012.73.834]

51. Kroenke K, Strine T, Spitzer R, Williams J, Berry J, Mokdad A. The PHQ-8 as a measure of current depression in the general population. J Affect Disord 2009 Apr;114(1-3):163-173. [doi: 10.1016/j.jad.2008.06.026] [Medline: 18752852]

52. Spitzer RL, Kroenke K, Williams JBW, Löwe B. A brief measure for assessing generalized anxiety disorder: the GAD-7. Arch Intern Med 2006 May 22;166(10):1092-1097. [doi: 10.1001/archinte.166.10.1092] [Medline: 16717171]

53. Kroenke K, Spitzer RL, Williams JBW. The Patient Health Questionnaire-2: validity of a two-item depression screener. Med Care 2003 Nov;41(11):1284-1292. [doi: 10.1097/01.MLR.0000093487.78664.3C] [Medline: 14583691]

54. Kroenke K, Spitzer RL, Williams JB, Monahan PO, Löwe B. Anxiety disorders in primary care: prevalence, impairment, comorbidity, and detection. Ann Intern Med 2007 Mar 06;146(5):317-325. [Medline: 17339617]

55. Brooke J. Usability evaluation in industry. In: Jordan PW, Weerdmeester BA, McClelland IL, editors. SUS-A quick dirty usability scale. Bristol, PA: Taylor \& Francis; 1996:107-114.

56. Bauermeister JA, Golinkoff JM, Muessig KE, Horvath KJ, Hightow-Weidman LB. Addressing engagement in technology-based behavioural HIV interventions through paradata metrics. Curr Opin HIV AIDS 2017 Sep;12(5):442-446. [doi: 10.1097/COH.0000000000000396] [Medline: 28617711]

57. Lenhart A. Pew Research Center. 2015 Apr 9. Teens, Social Media \& Technology Overview 2015 URL: http://www. pewinternet.org/2015/04/09/teens-social-media-technology-2015/ [accessed 2018-07-07] [WebCite Cache ID 70jXYM4ur]

58. Veinot TC, Meadowbrooke CC, Loveluck J, Hickok A, Bauermeister JA. How “community” matters for how people interact with information: mixed methods study of young men who have sex with other men. J Med Internet Res 2013 Feb 21;15(2):e33 [FREE Full text] [doi: 10.2196/jmir.2370] [Medline: 23428825]

59. Bauermeister JA, Leslie-Santana M, Johns MM, Pingel E, Eisenberg A. Mr. Right and Mr. Right Now: romantic and casual partner-seeking online among young men who have sex with men. AIDS Behav 2011 Feb;15(2):261-272 [FREE Full text] [doi: 10.1007/s10461-010-9834-5] [Medline: 20953689]

60. Winetrobe H, Rice E, Bauermeister J, Petering R, Holloway IW. Associations of unprotected anal intercourse with Grindr-met partners among Grindr-using young men who have sex with men in Los Angeles. AIDS Care 2014 Apr;26(10):1303-1308. [doi: 10.1080/09540121.2014.911811] [Medline: 24754563]

61. Norman CD, Skinner HA. eHEALS: The eHealth Literacy Scale. J Med Internet Res 2006 Nov;8(4):e27 [FREE Full text] [doi: 10.2196/jmir.8.4.e27] [Medline: 17213046 ]

62. Briggs A, Wonderling D, Mooney C. Pulling cost-effectiveness analysis up by its bootstraps: a non-parametric approach to confidence interval estimation. Health Econ 1997;6(4):327-340. [Medline: 9285227]

63. Patton M. Qualitative Research \& Evaluation Methods. Thousand Oaks, CA: Sage Publications; 2002.

64. Boyatzis R. Transforming Qualitative Information: Thematic Analysis and Code Development. Thousand Oaks, CA: Sage Publications; 1998:9780761909613.

65. Braun V, Clarke V. Using thematic analysis in psychology. Qualitative Research in Psychology 2006 Jan;3(2):77-101. [doi: 10.1191/1478088706qp063oa]

66. Luborsky M. The identification and analysis of themes and patterns. In: Gubrium J, Sankar A, editors. Qualitative methods in aging research. Thousand Oaks, CA: Sage Focus Editions; 1994:189-210.

67. Kubicek K, Arauz-Cuadra C, Kipke MD. Attitudes and perceptions of biomedical HIV prevention methods: voices from young men who have sex with men. Arch Sex Behav 2015 Feb;44(2):487-497 [FREE Full text] [doi: 10.1007/s10508-014-0398-8] [Medline: 25633499]

68. Buchbinder SP, Liu A. Pre-exposure prophylaxis and the promise of combination prevention approaches. AIDS Behav 2011 Apr;15 Suppl 1:S72-S79 [FREE Full text] [doi: 10.1007/s10461-011-9894-1] [Medline: 21331801]

69. THE BODY PRO. 2011. Pre-exposure prophylaxis (PrEP) for HIV prevention: Promoting safe and effective use in the United States URL: http://www.thebodypro.com/content/art59861.html [accessed 2017-05-22] [WebCite Cache ID 6qeplkkxX]

70. Grant RM, Lama JR, Anderson PL, McMahan V, Liu AY, Vargas L, et al. Preexposure chemoprophylaxis for HIV prevention in men who have sex with men. N Engl J Med 2010 Dec 30;363(27):2587-2599 [FREE Full text] [doi:

10.1056/NEJMoa1011205] [Medline: 21091279]

71. Wejnert C, Le B, Rose CE, Oster AM, Smith AJ, Zhu J, Gabriela Paz-Bailey for the NHBS Study Group. HIV infection and awareness among men who have sex with men-20 cities, United States, 2008 and 2011. PLoS One 2013 Oct;8(10):e76878 [FREE Full text] [doi: 10.1371/journal.pone.0076878] [Medline: 24194848] 
72. Hoots BE, Finlayson TJ, Wejnert C, Paz-Bailey G, NHBS Study Group. Early Linkage to HIV Care and Antiretroviral Treatment among Men Who Have Sex with Men--20 Cities, United States, 2008 and 2011. PLoS One 2015 Jul;10(7):e0132962 [FREE Full text] [doi: 10.1371/journal.pone.0132962] [Medline: 26176856]

73. Bauermeister JA, Eaton L, Andrzejewski J, Loveluck J, VanHemert W, Pingel ES. Where You Live Matters: Structural Correlates of HIV Risk Behavior Among Young Men Who Have Sex with Men in Metro Detroit. AIDS Behav 2015 Dec;19(12):2358-2369 [FREE Full text] [doi: 10.1007/s10461-015-1180-1] [Medline: 26334445]

74. Jeffries WL, Townsend ES, Gelaude DJ, Torrone EA, Gasiorowicz M, Bertolli J. HIV stigma experienced by young men who have sex with men (MSM) living with HIV infection. AIDS Educ Prev 2015 Feb;27(1):58-71. [doi: 10.1521/aeap.2015.27.1.58] [Medline: 25646730]

\author{
Abbreviations \\ ATOD: alcohol, tobacco, and other drug \\ CDC: Centers for Disease Control and Prevention \\ ES: effect sizes \\ GC: Get Connected \\ GLMM: generalized linear mixed models \\ IRB: institutional review board \\ LGBTQ: lesbian, gay, bisexual, transgender, questioning or queer \\ MSM: men who have sex with men \\ PrEP: pre-exposure prophylaxis \\ RCT: randomized controlled trial \\ SRV: subject recruitment venues \\ STI: sexually transmitted infection \\ YMSM: young men who have sex with men
}

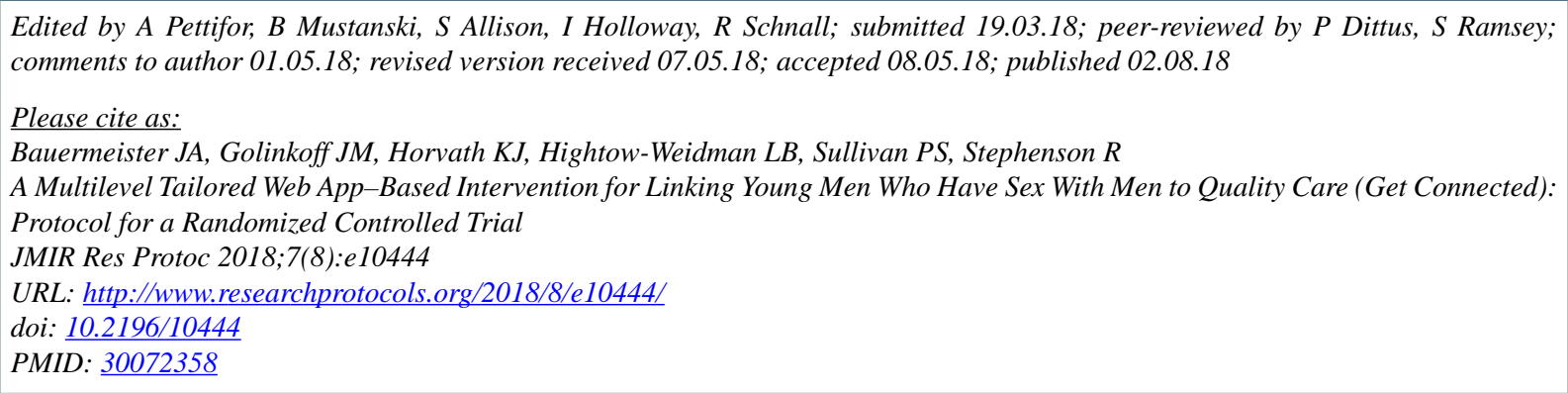

CJosé A. Bauermeister, Jesse M Golinkoff, Keith J Horvath, Lisa B Hightow-Weidman, Patrick S Sullivan, Rob Stephenson. Originally published in JMIR Research Protocols (http://www.researchprotocols.org), 02.08.2018. This is an open-access article distributed under the terms of the Creative Commons Attribution License (https://creativecommons.org/licenses/by/4.0/), which permits unrestricted use, distribution, and reproduction in any medium, provided the original work, first published in JMIR Research Protocols, is properly cited. The complete bibliographic information, a link to the original publication on http://www.researchprotocols.org, as well as this copyright and license information must be included. 